\title{
Auricular percutaneous nerve field stimulator device as alternative therapy for Cesarean delivery analgesia: proof of concept
}

\author{
Grace Lim, MD, MS (D) Kelsea R. LaSorda, MPH • Amy L. Monroe, MBA • \\ Jacques E. Chelly, MD, PhD, MBA
}

Received: 30 July 2019/Revised: 5 August 2019/Accepted: 6 August 2019/Published online: 20 August 2019

(c) Canadian Anesthesiologists' Society 2019

\section{To the Editor,}

Effective alternative pain therapies in the obstetric population are needed. Percutaneous nerve field stimulation (PNFS) has shown efficacy as a complementary method for postoperative pain management. ${ }^{1-3}$ In this pilot study, we assessed the feasibility and acceptability of the NSS2BRIDGE® (Innovative Health Solutions, Versailles, IN, USA) auricular PNFS device for post-Cesarean delivery analgesia.

After Institutional Review Board approval, written informed consent was obtained (ClinicalTrials.gov NCT03830307, date of registration: February 5 2019). Inclusion criteria were $\geq 18$ yr old, scheduled Cesarean delivery under single shot spinal anesthesia, and an American Society of Anesthesiologist physical status of II. Exclusion criteria were anxiety, active drug abuse, severe chronic pain, hemophilia, pacemaker, and psoriasis. Consecutive historical controls were identified from medical records of scheduled Cesarean deliveries and were matched by obstetrician and date of Cesarean delivery in the immediate four weeks prior to trial protocol initiation. Spinal anesthesia was induced per routine and

G. Lim, MD, MS ( $\varangle)$

Departments of Anesthesiology, Perioperative Medicine, Obstetrics \& Gynecology, University of Pittsburgh School of Medicine, Pittsburgh, PA, USA

e-mail: limkg2@upmc.edu

Magee-Womens Research Institute, Pittsburgh, PA, USA

K. R. LaSorda, MPH - A. L. Monroe, MBA ·

J. E. Chelly, MD, PhD, MBA

Department of Anesthesiology, Perioperative Medicine,

University of Pittsburgh School of Medicine, Pittsburgh, PA, USA no changes were made in standard postpartum analgesia regimens.

The NSS-2 BRIDGE $\AA$ device was fixed to one ear of the participant's choice by trained research staff immediately upon arrival to the postanesthesia care unit (Figure). Four leads were fixed with adhesive to the tragus, lobule, anterior scaphoid fossa, and posterior aspect of the helix. Training consisted of a five-minute video and inperson training session. The device remained fixed for 120 $\mathrm{hr}$ (five days) and was disposed of by the patient at home.

The primary endpoints of interest were pain at rest at one hour and $24 \mathrm{hr}$ postoperatively $(0-10$ numeric rating scale), and total opioid dose consumption during the 72-hr hospital stay (mg).

A total of five participants in the device group and five historical controls were included. Device tolerability was acceptable to patients. Pain scores at rest (one hour after the device was placed) were $68 \%$ lower in the device group than the control group (Figure). Pain scores at rest at $24 \mathrm{hr}$ were $72 \%$ lower in the device group. Oxycodone doses were 56\% higher in the control group (Figure).

In the device group, the range of pain scores with movement was 0-7 with one participant rating 10 at $48 \mathrm{hr}$, and the range of daily oxycodone at five days was $0-10 \mathrm{mg}$. Device discomfort scores were all 0 at $72 \mathrm{hr}$. There was no reported burning, buzzing, or site irritation. All devices were removed at home without complications. Minimal training was needed for staff to become proficient at device placement; proficiency was achieved after placing the device on patients twice under supervision.

These promising preliminary results reveal that the NSS2-BRIDGE ${ }^{\circledR}$ device exhibits high device tolerability by post-Cesarean delivery patients, minimal to no side effects, and marked reductions in pain at rest and inhospital opioid requirements. An ongoing investigation in a 


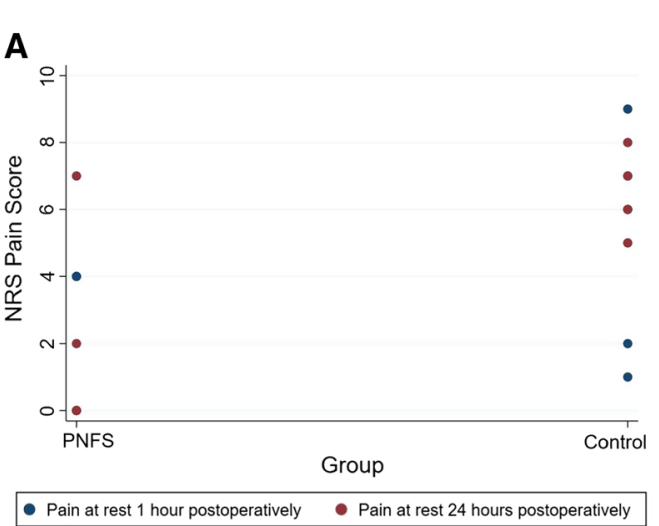

Figure Pain scores and opioid requirements between study groups. A) Pain scores at rest one hour after Cesarean delivery are presented from the PNFS and control groups (blue dots). Pain scores at rest 24 $\mathrm{hr}$ after Cesarean delivery are presented from the PNFS and control groups (red dots). B) Total dose requirements for oxycodone for the hospital stay ( $72 \mathrm{hr}$ ) were lower in the PNFS group than in the control

fully powered study sample that uses this study methodology is currently being organized (auricular PERcutaneous nerve field stimulator for Cesarean dEIIVEry trial, PERCEIVE). The PERCEIVE trial will provide definitive data to support or refute the use of auricular PNFS as an effective adjunct to post-Cesarean delivery multimodal analgesia.

Acknowledgement The authors are grateful to Innovative Solutions for the provision of the devices used in this study.

Conflicts of interest None declared.

Editorial responsibility This submission was handled by Dr. Philip M. Jones, Associate Editor, Canadian Journal of Anesthesia.

Funding Dr. Lim is supported in part by a grant from the NIH (K12HD043441). group. C) The NSS2-BRIDGE ${ }^{\circledR}$ (Innovative Health Solutions, Versailles, IN, USA) device applied to a participant ear. Electrodes minimally penetrate dermis and are affixed with adhesive. The primary unit is affixed behind the ear with adhesive. PNFS = percutaneous nerve field stimulator. NRS $=$ numeric rating scale pain score.

\section{References}

1. Babygirija R, Sood M, Kannampalli P, Sengupta JN, Miranda A. Percutaneous electrical nerve field stimulation modulates central pain pathways and attenuates post-inflammatory visceral and somatic hyperalgesia in rats. Neuroscience 2017; 356: 11-21.

2. Kovacic K, Hainsworth $K$, Sood M, et al. Neurostimulation for abdominal pain-related functional gastrointestinal disorders in adolescents: a randomised, double-blind, sham-controlled trial. Lancet Gastroenterol Hepatol 2017; 2: 727-37.

3. Miranda A, Taca A. Neuromodulation with percutaneous electrical nerve field stimulation is associated with reduction in signs and symptoms of opioid withdrawal: a multisite, retrospective assessment. Am J Drug Alcohol Abuse 2018; 44: 56-63.

Publisher's Note Springer Nature remains neutral with regard to jurisdictional claims in published maps and institutional affiliations. 